\title{
Tailored flower pollination (TFP) algorithm for diminution of real power loss
}

\author{
K.Lenin \\ Department of EEE, Prasad V.Potluri Siddhartha Institute of Technology, India
}

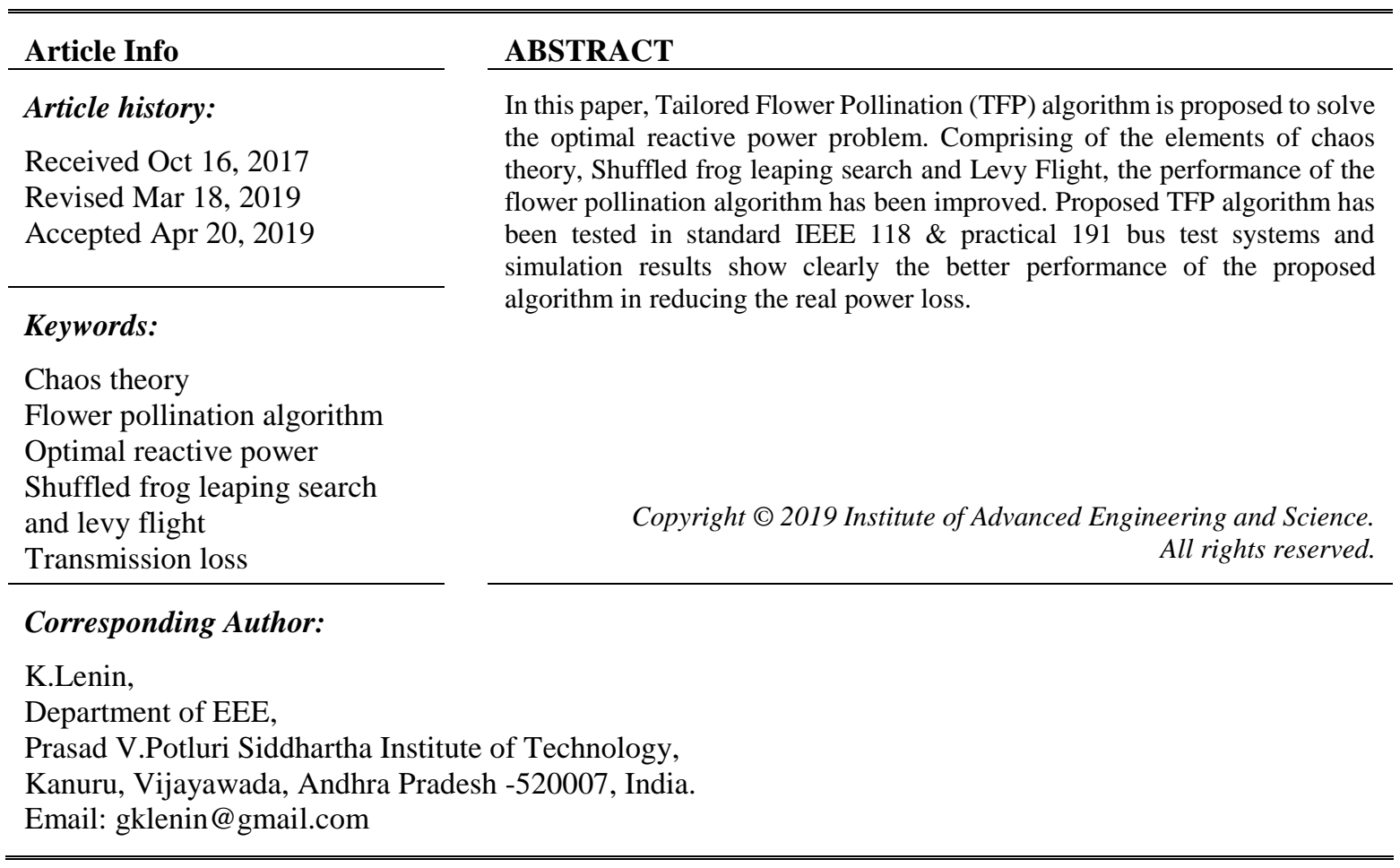

\section{INTRODUCTION}

Optimal reactive power dispatch (ORPD) problem is to minimize the real power loss and bus voltage deviation. Various numerical methods like the gradient method [1-2], Newton method [3] and linear programming [4-7] have been adopted to solve the optimal reactive power dispatch problem. Both the gradient and Newton methods have the complexity in managing inequality constraints. If linear programming is applied then the input- output function has to be uttered as a set of linear functions which mostly lead to loss of accuracy. The problem of voltage stability and collapse play a major role in power system planning and operation [8]. Evolutionary algorithms such as genetic algorithm have been already proposed to solve the reactive power flow problem [9-11]. Evolutionary algorithm is a heuristic approach used for minimization problems by utilizing nonlinear and non-differentiable continuous space functions. In [12], Hybrid differential evolution algorithm is proposed to improve the voltage stability index. In [13] Biogeography Based algorithm is projected to solve the reactive power dispatch problem. In [14], a fuzzy based method is used to solve the optimal reactive power scheduling method. In [15], an improved evolutionary programming is used to solve the optimal reactive power dispatch problem. In [16], the optimal reactive power flow problem is solved by integrating a genetic algorithm with a nonlinear interior point method. In [17], a pattern algorithm is used to solve ac-dc optimal reactive power flow model with the generator capability limits. In [18], F. Capitanescu proposes a two-step approach to evaluate Reactive power reserves with respect to operating constraints and voltage stability. In [19], a programming based approach is used to solve the optimal reactive power dispatch problem. In [20], A. Kargarian et al present a probabilistic algorithm for optimal reactive power provision in hybrid electricity markets with uncertain loads. This paper proposes Tailored Flower Pollination (TFP) algorithm is proposed to solve the reactive power problem. The basic idea of flower pollination process which leads to the formulation of flower pollination algorithm (FPA) [21] is first introduced and subsequently, chaos 
theory, Shuffled frog leaping search and Levy Flight are introduced. Proposed TFP algorithm has been evaluated in standard IEEE 118 \& practical 191 bus test systems. Simulation results show that our proposed approach outperforms all the entitled reported algorithms in minimization of real power loss.

\section{PROBLEM FORMULATION}

The optimal power flow problem is treated as a general minimization problem with constraints, and can be mathematically written in the following form:

$$
\text { Minimize } f(x, u)
$$

subject to

$$
\mathrm{g}(\mathrm{x}, \mathrm{u})=0
$$

and

$$
\mathrm{h}(\mathrm{x}, \mathrm{u}) \leq 0
$$

where $f(x, u)$ is the objective function. $g(x . u)$ and $h(x, u)$ are respectively the set of equality and inequality constraints. $\mathrm{x}$ is the vector of state variables, and $\mathrm{u}$ is the vector of control variables.

The state variables are the load buses (PQ buses) voltages, angles, the generator reactive powers and the slack active generator power:

$$
x=\left(P_{g 1}, \theta_{2}, \ldots, \theta_{N}, V_{L 1}, ., V_{L N L}, Q_{g 1}, \ldots, Q_{g n g}\right)^{T}
$$

The control variables are the generator bus voltages, the shunt capacitors/reactors and the transformers tapsettings:

$$
\mathrm{u}=\left(\mathrm{V}_{\mathrm{g}}, \mathrm{T}, \mathrm{Q}_{\mathrm{c}}\right)^{\mathrm{T}}
$$

or

$$
\mathrm{u}=\left(\mathrm{V}_{\mathrm{g} 1}, \ldots, \mathrm{V}_{\mathrm{gng}}, \mathrm{T}_{1}, \ldots, \mathrm{T}_{\mathrm{Nt}}, \mathrm{Q}_{\mathrm{c} 1}, \ldots, \mathrm{Q}_{\mathrm{cNc}}\right)^{\mathrm{T}}
$$

where ng, nt and nc are the number of generators, number of tap transformers and the number of shunt compensators respectively.

\section{OBJECTIVE FUNCTION}

\subsection{Active power loss}

The objective of the reactive power dispatch is to minimize the active power loss in the transmission network, which can be described as follows:

$$
F=P L=\sum_{k \in N b r} g_{k}\left(V_{i}^{2}+V_{j}^{2}-2 V_{i} V_{j} \cos \theta_{i j}\right)
$$

or

$$
F=P L=\sum_{i \in N g} P_{g i}-P_{d}=P_{g s l a c k}+\sum_{i \neq s l a c k}^{N g} P_{g i}-P_{d}
$$

where $g_{k}$ : is the conductance of branch between nodes $\mathrm{i}$ and $\mathrm{j}$, Nbr: is the total number of transmission lines in power systems. $\mathrm{P}_{\mathrm{d}}$ : is the total active power demand, $\mathrm{P}_{\mathrm{gi}}$ : is the generator active power of unit $\mathrm{i}$, and $\mathrm{P}_{\mathrm{gsalck}}$ : is the generator active power of slack bus.

\subsection{Voltage profile improvement}

For minimizing the voltage deviation in PQ buses, the objective function becomes:

$$
F=P L+\omega_{v} \times V D
$$


where $\omega_{\mathrm{v}}$ : is a weighting factor of voltage deviation. VD is the voltage deviation given by:

$$
V D=\sum_{i=1}^{N p q}\left|V_{i}-1\right|
$$

\subsection{Equality Constraint}

The equality constraint $\mathrm{g}(\mathrm{x}, \mathrm{u})$ of the ORPD problem is represented by the power balance equation, where the total power generation must cover the total power demand and the power losses:

$$
P_{G}=P_{D}+P_{L}
$$

This equation is solved by running Newton Raphson load flow method, by calculating the active power of slack bus to determine active power loss.

\subsection{Inequality Constraints}

The inequality constraints $\mathrm{h}(\mathrm{x}, \mathrm{u})$ reflect the limits on components in the power system as well as the limits created to ensure system security. Upper and lower bounds on the active power of slack bus, and reactive power of generators:

$$
\begin{aligned}
& P_{\text {gslack }}^{\min } \leq P_{\text {gslack }} \leq P_{\text {gslack }}^{\max } \\
& Q_{g i}^{\min } \leq Q_{g i} \leq Q_{g i}^{\max }, i \in N_{g}
\end{aligned}
$$

Upper and lower bounds on the bus voltage magnitudes:

$$
V_{i}^{\min } \leq V_{i} \leq V_{i}^{\max }, i \in N
$$

Upper and lower bounds on the transformers tap ratios:

$$
T_{i}^{\min } \leq T_{i} \leq T_{i}^{\max }, i \in N_{T}
$$

Upper and lower bounds on the compensators reactive powers:

$$
Q_{c}^{\min } \leq Q_{c} \leq Q_{C}^{\max }, i \in N_{C}
$$

where $\mathrm{N}$ is the total number of buses, $\mathrm{N}_{\mathrm{T}}$ is the total number of Transformers; $\mathrm{N}_{\mathrm{c}}$ is the total number of shunt reactive compensators.

\section{FLOWER POLLINATION ALGORITHM}

Generally we use the following systems in Flower Pollination Algorithm (FPA),

- System 1. Biotic and cross-pollination has been treated as global pollination process, and pollen-carrying pollinators travel in a way which obeys Levy flights.

- System 2. For local pollination, A- biotic and self-pollination has been utilized.

- System 3. Pollinators such as insects can develop flower reliability, which is equivalent to a reproduction probability and it is proportional to the similarity of two flowers implicated.

- System 4. The communication of local pollination and global pollination can be controlled by a control probability $p \in[0,1]$, with a slight bias towards local pollination.

System 1 and flower reliability can be represented mathematically as

$$
x_{i}^{t+1}=x_{i}^{t}+\gamma L(\lambda)\left(x_{i}^{t}-g_{*}\right)
$$

where $x_{i}^{t}$ is the pollen $i$ or solution vector $x_{i}$ at iteration $t$, and $g_{*}$ is the current best solution found among all solutions at the current generation/iteration. Here $\gamma$ is a scaling factor to control the step size. $L(\lambda)$ is the parameter that corresponds to the strength of the pollination, which essentially is also the step size. Since insects may move over a long distance with various distance steps, we can use a Levy flight to mimic this characteristic efficiently. We draw $L>0$ from a Levy distribution

$$
L \sim \frac{\lambda \Gamma(\lambda \sin (\Pi \lambda / 2))}{\Pi} \frac{1}{s^{1+\lambda}},\left(s \gg s_{0}>0\right)
$$


here, $\Gamma(\lambda)$ is the standard gamma function, and this distribution is valid for large steps $s>0$. Then, to model the local pollination, for both system 2 and system 3 can be represented as

$$
x_{i}^{t+1}=x_{i}^{t}+\in\left(x_{j}^{t}-x_{k}^{t}\right)
$$

where $x_{j}^{t}$ and $x_{k}^{t}$ are pollen from different flowers of the same plant species. This essentially mimics the flower reliability in a limited neighbourhood. Mathematically, if $x_{j}^{t}$ and $x_{k}^{t}$ comes from the same species or selected from the same population, this equivalently becomes a local random walk if we draw $\in$ from a uniform distribution in $[0,1]$. Though Flower pollination performance can occur at all balance, local and global, neighbouring flower patch or flowers in the not-so-far-away neighbourhood are more likely to be pollinated by local flower pollen than those far away. In order to mimic this, we can effectively use a control probability (system 4) or proximity probability $p$ to switch between common global pollination to intensive local pollination. To start with, we can use a raw value of $p=0.8$ as an initially value.

The simplest method is to use a weighted sum to combine all multiple objectives into a composite single objective

$$
f=\sum_{i=1}^{m} w_{i} f_{i} \sum_{i=1}^{m} w_{i}=1, w_{i}>0
$$

where $m$ is the number of objectives and $\mathrm{w}_{\mathrm{i}}(\mathrm{i}=1, \ldots, \mathrm{m})$ are non-negative weights.

FP Algorithm for solving optimal reactive power optimization

Step 1. Objective min of $(x), x=\left(x_{1}, x_{2}, \ldots, x_{d}\right)$

Step 2. Initialize a population of $n$ flowers

Step 3. Find the best solution $g_{*}$ in the initial population

Step 4. Define a control probability $p \in[0,1]$

Step5. Define a stopping criterion (a fixed number of generations/iterations)

Step6. while ( $t<$ Max Generation)

Step6. for $i=1: n$ (all $n$ flowers in the population)

Step7. if rand $<p$,

Step8. Draw a (d-dimensional) step vector $L$ which obeys a Levy distribution Global pollination through $x_{i}^{t+1}=x_{i}^{t}+L\left(x_{i}^{t}-g_{*}\right)$

else

step9. Draw $\in$ from a uniform distribution in $[0,1]$

step 10.Do local pollination through $x_{i}^{t+1}=x_{i}^{t}+\in\left(x_{j}^{t}-x_{k}^{t}\right)$

end if

step 10. Evaluate new solutions

step11. If new solutions are better, update them in the population

end for

step12. Find the current best solution $g_{*}$

end while

Output - best solution has been found

\section{CHAOTIC MAPS}

Chaos is a random state found in the non-linear dynamical deterministic system, possesses non-period, non-converging and bounded properties. The use of chaotic sequences is more beneficial than the random sequences due to its non-repetition and ergodicity properties. Borrowing the advantages of ergodicity, nonrepetition and randomness of the chaotic sequences, the chaotic map is replacing the random sequences in generating the initial population in the FPA in this study. This is to ensure that the diversity of the initial population can be improved, where the distribution of the initial population is more uniform. Ten different chaotic maps are and circle map is selected for the integration with FPA.

$$
x_{n+1}=\left(x_{n}+0.2-(0.5 / 2 \pi) \sin \left(2 \pi x_{n}\right)\right) \times \bmod (1)
$$

\section{SHUFFLED FROG LEAPING ALGORITHM}

Shuffled frog leaping algorithm is a biological evolution algorithm based on swarm intelligence. The algorithm simulates a group of frogs in the wetland passing thought and foraging by classification of ethnic 
groups. In the execution of the algorithm, $\mathrm{F}$ frogs are generated at first to form a group, for $\mathrm{N}$-dimensional optimization problem, frog $\mathrm{i}$ of the group is represented as $X_{i}=\left(x_{i}^{1}, x_{i}^{2}, \ldots, x_{i}^{N}\right)$ then individual frogs in the group are sorted in descending order according to fitness values, to find the global best solution $\mathrm{P}_{\mathrm{x}}$. The group is divided into $m$ ethnic groups, each ethnic group including $n$ frogs, satisfying the relation $F=m \times n$. The rule of ethnic group division is: the first frog into the first sub-group, the second frog into the second sub-group, frog $m$ into sub-group $m$, frog $m+1$ into the first sub-group again, frog $m+2$ into the second sub-group, and so on, until all the frogs are divided, then find the best frog in each sub-group, denoted by $\mathrm{P}_{\mathrm{b}}$; get a worst frog correspondingly, denoted by $\mathrm{P}_{\mathrm{w}}$. Its iterative formula can be expressed as:

$$
\begin{aligned}
& D=\operatorname{rand}() *\left(P_{b}-P_{\omega}\right) \\
& P_{\text {new_- } \omega}=P_{\omega}+D_{i},-D_{\max } \leq D_{i} \leq D_{\max }
\end{aligned}
$$

where rand() represents a random number between 0 and $1, \mathrm{P}_{\mathrm{b}}$ represents the position of the best frog, $\mathrm{P}_{\mathrm{w}}$ represents the position of the worst frog, D represents the distance moved by the worst frog, $P_{n e w_{-}-\omega}$ is the improved position of the frog, $\mathrm{D}_{\max }$ represents the step length of frog leaping.

In the execution of the algorithm, if the updated $P_{n e w_{-}-\omega}$ is in the feasible solution space, calculate the corresponding fitness value of $P_{n e w_{-}-\omega}$, if the corresponding fitness value of $P_{n e w_{-}-\omega}$ is worse than the corresponding fitness value of $\mathrm{P}_{\mathrm{w}}$, then use $\mathrm{P}_{\mathrm{w}}$ to replace $\mathrm{P}_{\mathrm{b}}$ in equation (22) and re-update $P_{n e w_{-}-\omega}$; if there is still no improvement, then randomly generate a new frog to replace $\mathrm{P}_{\mathrm{w}}$; repeat the update process until satisfying stop conditions.

\section{LEVY FLIGHT}

Levy flight is a rank of non-Gaussian random processes whose arbitrary walks are drawn from Levy stable distribution. This allocation is a simple power-law formula $L(s) \sim|s|^{-1-\beta}$ where $0<\beta<2$ is an index. Mathematically exclamation, a easy version of Levy distribution can be defined as ,

$$
L(s, \gamma, \mu)=\left\{\begin{array}{c}
\sqrt{\frac{\gamma}{2 \pi}} \\
0 \text { if } s \leq 0
\end{array} \exp \left[-\frac{\gamma}{2(s-\mu)}\right] \frac{1}{(s-\mu)^{3 / 2}} \text { if } 0<\mu<s<\infty\right.
$$

where $\gamma>0$ parameter is scale (controls the scale of distribution) parameter, $\mu$ parameter is location or shift parameter. In general, Levy distribution should be defined in terms of Fourier transform

$$
F(k)=\exp \left[-\alpha|k|^{\beta}\right], 0<\beta \leq 2,
$$

where $\alpha$ is a parameter within [-1,1] interval and known as scale factor. An index of o stability $\beta \in[0,2]$ is also referred to as Levy index. In particular, for $\beta=1$, the integral can be carried out analytically and is known as the Cauchy probability distribution. One more special case when $\beta=2$, the distribution correspond to Gaussian distribution. $\beta$ and $\alpha$ parameters take a key part in determination of the distribution. The parameter $\beta$ controls the silhouette of the probability distribution in such a way that one can acquire different shapes of probability distribution, especially in the tail region depending on the parameter $\beta$. Thus, the smaller $\beta$ parameter causes the distribution to make longer jumps since there will be longer tail. It makes longer jumps for smaller values whereas it makes shorter jumps for bigger values. By Levy flight, new-fangled state of the particle is designed as,

$$
X^{t+1}=X^{t}+\alpha \oplus \operatorname{Levy}(\beta)
$$

$\alpha$ is the step size which must be related to the scales of the problem of interest. In the proposed method $\alpha$ is random number for all dimensions of particles.

$$
X^{t+1}=X^{t}+\operatorname{random}(\operatorname{size}(D)) \oplus \operatorname{Levy}(\beta)
$$

the product $\bigoplus$ means entry-wise multiplications.

A non-trivial scheme of generating step size s samples are summarized as follows,

$$
X^{t+1}=X^{t}+\operatorname{random}(\operatorname{size}(D)) \oplus \operatorname{Levy}(\beta) \sim 0.01 \frac{u}{|v|^{1 / \beta}}\left(x_{j}^{t}-g b\right)
$$


where $u$ and $v$ are drawn from normal distributions. That is

$$
u \sim N\left(0, \sigma_{u}^{2}\right) v \sim N\left(0, \sigma_{v}^{2}\right)
$$

with

$$
\sigma_{u}=\left\{\frac{\Gamma(1+\beta) \sin (\pi \beta / 2)}{\Gamma[(1+\beta) / 2] \beta 2^{(\beta-1) / 2}}\right\}^{1 / \beta}, \sigma_{v}=1
$$

here $\Gamma$ is standard Gamma function. One of the important points to be considered while performing distribution by Levy flights is the value taken by the $\beta$ parameter and it substantially affects distribution.

\section{TAILORED FLOWER POLLINATION (TFP) ALGORITHM}

In the TFP algorithm, the initial population is generated using the circle map, frog leaping local search is performed by each solution and when rand $>p$, modified Levy flight with integration of inertia weight in global pollination is performed on that particular solution. The steps involved in the TFP are as follows:

Step 1.Parameter Initialization: Initialize the relevant parameters of population size, $n$, dimension of search space, $d$, maximum iteration, max_iter, switch probability, $p$, range of search space $[L b, U b m]$, number of memeplexes, $\mathrm{m}$ and iterations within each memeplex, $i t$.

Step 2.create Initial Population using the chaotic map

Step 3.Find the Best Solution: The fitness value of each solution is calculated and the best solution is determined.

Step 4.Perform the Frog Leaping Search - For each solution, search is performed.

Step 5.Perform the Global Search of Flower Pollination Algorithm

Step 6.Update the Solution: The fitness value of each new solution is evaluated. The historical position is updated through comparison with the new solution. Subsequently, the best solution is updated.

Step 7.Check Termination Condition or else, Step 4 is repeated.

\section{SIMULATION RESULTS}

At first Tailored Flower Pollination (TFP) algorithm has been tested in standard IEEE 118-bus test system [22].The system has 54 generator buses, 64 load buses, 186 branches and 9 of them are with the tap setting transformers. The limits of voltage on generator buses are $0.95-1.1$ per-unit., and on load buses are $0.95-1.05$ per-unit. The limit of transformer rate is $0.9-1.1$, with the changes step of 0.025 . The limitations of reactive power source are listed in Table 1 , with the change in step of 0.01 .

Table 1. Limitation of Reactive Power Sources

\begin{tabular}{cccccccc}
\hline BUS & 5 & 34 & 37 & 44 & 45 & 46 & 48 \\
\hline QCMAX & 0 & 14 & 0 & 10 & 10 & 10 & 15 \\
QCMIN & -40 & 0 & -25 & 0 & 0 & 0 & 0 \\
BUS & 74 & 79 & 82 & 83 & 105 & 107 & 110 \\
QCMAX & 12 & 20 & 20 & 10 & 20 & 6 & 6 \\
QCMIN & 0 & 0 & 0 & 0 & 0 & 0 & 0 \\
\hline
\end{tabular}

The statistical comparison results of 50 trial runs have been list in Table 2 and the results clearly show the better performance of proposed TFP algorithm.

Table 2. Comparison Results

\begin{tabular}{ccccc}
\hline Active power loss (p.u) & BBO [23] & ILSBBO/strategy1 [23] & ILSBBO/strategy1 [23] & Proposed TFP \\
\hline Min & 128.77 & 126.98 & 124.78 & 116.02 \\
Max & 132.64 & 137.34 & 132.39 & 119.12 \\
Average & 130.21 & 130.37 & 129.22 & 117.32 \\
\hline
\end{tabular}

Then the Tailored Flower Pollination (TFP) algorithm has been tested in practical 191 test system and the following results have been obtained. In Practical 191 test bus system - Number of Generators $=20$, Number of lines $=200$, Number of buses $=191$ Number of transmission lines $=55$. Table 3 shows the optimal 
control values of practical 191 test system obtained by TFP method. And table 4 shows the results about the value of the real power loss by obtained by Tailored Flower Pollination (TFP) algorithm.

Table 3. Optimal Control Values of Practical 191 Utility (Indian) System by TFP Method

\begin{tabular}{cccc}
\hline VG1 & 1.10 & VG 11 & 0.90 \\
\cline { 4 - 4 } VG 2 & 0.78 & VG 12 & 1.00 \\
VG 3 & 1.01 & VG 13 & 1.00 \\
VG 4 & 1.01 & VG 14 & 0.90 \\
VG 5 & 1.10 & VG 15 & 1.00 \\
VG 6 & 1.10 & VG 16 & 1.00 \\
VG 7 & 1.10 & VG 17 & 0.90 \\
VG 8 & 1.01 & VG 18 & 1.00 \\
VG 9 & 1.10 & VG 19 & 1.10 \\
VG 10 & 1.01 & VG 20 & 1.10 \\
\hline
\end{tabular}

\begin{tabular}{ll}
\hline T1 & 1.00 \\
\hline $\mathrm{T} 2$ & 1.00 \\
$\mathrm{~T} 3$ & 1.00 \\
$\mathrm{~T} 4$ & 1.10 \\
$\mathrm{~T} 5$ & 1.00 \\
$\mathrm{~T} 6$ & 1.00 \\
$\mathrm{~T} 7$ & 1.00 \\
$\mathrm{~T} 8$ & 1.01 \\
$\mathrm{~T} 9$ & 1.00 \\
$\mathrm{~T} 10$ & 1.00 \\
$\mathrm{~T} 11$ & 0.90 \\
$\mathrm{~T} 12$ & 1.00 \\
$\mathrm{~T} 13$ & 1.01 \\
$\mathrm{~T} 14$ & 1.01 \\
$\mathrm{~T} 15$ & 1.01 \\
$\mathrm{~T} 19$ & 1.02 \\
$\mathrm{~T} 20$ & 1.01 \\
\hline
\end{tabular}

\begin{tabular}{ll}
\hline T21 & 0.90 \\
\hline T22 & 0.90 \\
T23 & 0.90 \\
T24 & 0.90 \\
T25 & 0.90 \\
T26 & 1.00 \\
T27 & 0.90 \\
T28 & 0.90 \\
T29 & 1.01 \\
T30 & 0.90 \\
T31 & 0.90 \\
T32 & 0.90 \\
T33 & 1.01 \\
T34 & 0.90 \\
T35 & 0.90 \\
T39 & 0.90 \\
T40 & 0.90 \\
\hline
\end{tabular}

\begin{tabular}{ll}
\hline T41 & 0.90 \\
\hline T42 & 0.90 \\
T43 & 0.91 \\
T44 & 0.91 \\
T45 & 0.91 \\
T46 & 0.90 \\
T47 & 0.91 \\
T48 & 1.00 \\
T49 & 0.90 \\
T50 & 0.90 \\
T51 & 0.90 \\
T52 & 0.90 \\
T53 & 1.00 \\
T54 & 0.90 \\
T55 & 0.90 \\
\hline
\end{tabular}

Table 4. Optimum Real Power Loss Values Obtained for Practical 191 Utility (Indian) System by TFP Method

\begin{tabular}{lc}
\hline Real power Loss (MW) & TFP \\
\hline Min & 144.982 \\
Max & 147.992 \\
Average & 145.832 \\
\hline
\end{tabular}

\section{CONCLUSION}

Tailored Flower Pollination (TFP) algorithm has been effectively applied for solving reactive power problem. And it has been tested in standard IEEE 118 \& practical 191 bus test systems. Performance comparisons with well-known population-based algorithms give improved results. Tailored Flower Pollination (TFP) algorithm emerges to find good solutions when compared to that of other reported algorithms. The simulation results presented in previous section prove the capability of TFP approach to arrive at near to global optimal solution.

\section{REFERENCES}

[1] O. Alsac and B. Stott, "Optimal Load Flow with Steady-State Security," in IEEE Transactions on Power Apparatus and Systems, vol. PAS-93, no. 3, pp. 745-751, May 1974.

[2] K. Y. Lee, Y. M. Park and J. L. Ortiz, "A United Approach to Optimal Real and Reactive Power Dispatch," in IEEE Transactions on Power Apparatus and Systems, vol. PAS-104, no. 5, pp. 1147-1153, May 1985.

[3] A. Monticelli, M. V. F. Pereira and S. Granville, "Security-Constrained Optimal Power Flow with Post-Contingency Corrective Rescheduling," in IEEE Transactions on Power Systems, vol. 2, no. 1, pp. 175-180, Feb. 1987.

[4] N. Deeb and S. M. Shahidehpour, "Linear reactive power optimization in a large power network using the decomposition approach," in IEEE Transactions on Power Systems, vol. 5, no. 2, pp. 428-438, May 1990.

[5] E. Hcbson, "Network Constrained Reactive Power Control Using Linear Programming," in IEEE Transactions on Power Apparatus and Systems, vol. PAS-99, no. 3, pp. 868-877, May 1980.

[6] K. Y. Lee, Y. M. Park and J. L. Ortiz, "Fuel-cost minimisation for both real-and reactive-power dispatches," in IEE 
Proceedings C - Generation, Transmission and Distribution, vol. 131, no. 3, pp. 85-93, May 1984.

[7] M.K. Mangoli, and K.Y. Lee, "Optimal real and reactive power control using linear programming", Electr. Power Syst. Res., vol. 26, no. 1, pp.1-10, January 1993.

[8] C. A. Canizares, A. C. Z. De Souza and V. H. Quintana, "Comparison of performance indices for detection of proximity to voltage collapse," in IEEE Transactions on Power Systems, vol. 11, no. 3, pp. 1441-1450, Aug. 1996.

[9] S.R.Paranjothi, and K.Anburaja, "Optimal power flow using refined genetic algorithm", Electr. Power Compon. Syst, vol. 30, no. 10, pp. 1055-1063, 2002.

[10] D. Devaraj and B. Yegnanarayana, "Genetic-algorithm-based optimal power flow for security enhancement," in IEE Proceedings - Generation, Transmission and Distribution, vol. 152, no. 6, pp. 899-905, 4 Nov. 2005.

[11] A. Berizzi, C. Bovo, M. Merlo, and M. Delfanti, "A GA approach to compare orpf objective functions including secondary voltage regulation," Electric Power Systems Research, vol. 84, no. 1, pp. 187 - 194, 2012.

[12] C.-F. Yang, G. G. Lai, C.-H. Lee, C.-T. Su, and G. W. Chang, "Optimal setting of reactive compensation devices with an improved voltage stability index for voltage stability enhancement," International Journal of Electrical Power and Energy Systems, vol. 37, no. 1, pp. 50 - 57, 2012.

[13] P. Roy, S. Ghoshal, and S. Thakur, "Optimal var control for improvements in voltage profiles and for real power loss minimization using biogeography based optimization," International Journal of Electrical Power and Energy Systems, vol. 43, no. 1, pp. $830-838,2012$.

[14] B. Venkatesh, G. Sadasivam and M. A. Khan, "A new optimal reactive power scheduling method for loss minimization and voltage stability margin maximization using successive multi-objective fuzzy LP technique," in IEEE Transactions on Power Systems, vol. 15, no. 2, pp. 844-851, May 2000.

[15] Wei Yan, Shuai Lu and D. C. Yu, "A novel optimal reactive power dispatch method based on an improved hybrid evolutionary programming technique," in IEEE Transactions on Power Systems, vol. 19, no. 2, pp. 913-918, May 2004.

[16] Wei Yan, Fang Liu, C. Y. Chung and K. P. Wong, "A hybrid genetic algorithm-interior point method for optimal reactive power flow," in IEEE Transactions on Power Systems, vol. 21, no. 3, pp. 1163-1169, Aug. 2006.

[17] J. Yu, W. Yan, W. Li, C. Y. Chung and K. P. Wong, "An Unfixed Piecewise-Optimal Reactive Power-Flow Model and its Algorithm for AC-DC Systems," in IEEE Transactions on Power Systems, vol. 23, no. 1, pp. 170-176, Feb. 2008.

[18] F. Capitanescu, "Assessing Reactive Power Reserves With Respect to Operating Constraints and Voltage Stability," in IEEE Transactions on Power Systems, vol. 26, no. 4, pp. 2224-2234, Nov. 2011.

[19] Z. Hu, X. Wang, and G. Taylor, "Stochastic optimal reactive power dispatch: Formulation and solution method," International Journal of Electrical Power and Energy Systems, vol. 32, no. 6, pp. 615 - 621, July 2010.

[20] A. Kargarian, M. Raoofat, and M. Mohammadi, "Probabilistic reactive power procurement in hybrid electricity markets with uncertain loads," Electric Power Systems Research, vol. 82, no. 1, pp. 68 - 80, January 2012.

[21] Yang, X. S. "Flower pollination algorithm for global optimization, in: Unconventional Computation and Natural Computation, ” In: Durand-Lose J., Jonoska N. (eds) Unconventional Computation and Natural Computation. UCNC 2012. Lecture Notes in Computer Science, Vol. 7445, pp. 240-249, 2012.

[22] IEEE, "The IEEE 30-bus test system and the IEEE 118-test system”, (1993), $\mathrm{http}: / /$ www.ee.washington.edu/trsearch/pstca/.

[23] Jiangtao Cao, Fuli Wang and Ping Li, "An Improved Biogeography-based Optimization Algorithm for Optimal Reactive Power Flow", International Journal of Control and Automation, vol.7, no.3 , pp.161-176, March 2014. 